\title{
ABELIAN DIFFERENTIALS WITH DOUBLE ZEROS
}

\section{HERSHEL M. FARKAS ${ }^{1}$}

\begin{abstract}
In this note we give a simple proof of the following theorem: The locus of points in the Torelli space of compact Riemann surfaces of genus $g \geqq 2$ whose underlying surfaces do not permit a basis for the abelian differentials of first kind each of whose elements is a differential with double zeros, has positive codimension in the Torelli space.
\end{abstract}

Introduction. One of the questions which seems to have occupied people in the late nineteenth century is the question of the existence of a basis for the abelian differentials of first kind on a compact Riemann surface of genus $g$ each of whose elements consists of a differential with double zeros. It would seem as though this question has never been answered. Here we prove the following theorem: The locus of points in the Torelli space of compact Riemann surfaces of genus $g$ whose underlying surfaces do not permit a basis for the abelian differentials of first kind each of whose elements is a differential with double zeros, has positive codimension in the Torelli space. In other words, using the language of [F1], the property of not permitting such a basis is special in the sense of moduli.

The proof of this theorem follows quite simply from a slight generalization of a theorem of Lewittes [ $L$, Theorem 12] suggested by Accola during a seminar talk given by the author on this question and ideas in [R]. The author would like to thank Professor H. E. Rauch for bringing this question to his attention and also for his many helpful suggestions.

If $S$ is a compact Riemann surface of genus $g \geqq 2$ with canonical homology basis $(\Gamma, \Delta), \Gamma=\gamma_{1}, \cdots, \gamma_{g}, \Delta=\delta_{1}, \cdots, \delta_{g}$, and $\omega_{1}, \cdots$, $\omega_{0}$ is the normal basis for the space of abelian differentials of first kind on $S$ with respect to $\Gamma, \Delta$ i.e.: $\int_{\gamma_{j}} \omega_{i}=\delta_{i j}$, we obtain a $g \times g$ symmetric matrix

$$
\Pi=\left(\Pi_{i j}\right) \quad \text { where } \quad \Pi_{i j}=\int_{\delta_{j}} \omega_{i} \text { and } \operatorname{Im} \Pi \gg 0 .
$$

The collection of all such matrices, $g \times g$ symmetric with positive

Received by the editors February 19, 1970.

AMS 1970 subject classifications. Primary 30A52.

Key words and phrases. Abelian differentials, theta functions, Riemann surfaces moduli.

${ }^{1}$ Research partially supported by NSF GP 12467. 
definite imaginary part is called the Siegel upper half plane of degree $g$ and is denoted by $\mathfrak{S}_{g}$. The $g \times 2 g$ matrix $(I I)$ where $I$ is the $g \times g$ identity matrix is called the period matrix for $\omega_{1}, \cdots, \omega_{0}$, and the space of abelian differentials of first kind will be denoted by $A_{1}$.

The Riemann theta function

$$
\theta(\zeta, \Pi)=\sum_{N \in Z^{\sigma}} \exp 2 \Pi i\left[\frac{1}{2} N \Pi N+N \zeta\right],
$$

where $\zeta \in C^{\sigma}, N$ an integer vector in $C^{o}$ and $\Pi \in \Im_{g}$ is a holomorphic function on $C^{o} \times \mathfrak{S}_{\rho}$ and in particular for a fixed $\Pi \in \mathfrak{S}_{\rho}$, is an entire function on $\boldsymbol{C}^{a}$.

Given a compact Riemann surface and $\Pi$ constructed as above, we may view the Riemann theta function as a multiplicative function on the Riemann surface $S$. More specifically, we choose a point $p_{0} \in S$ and consider the map $\phi^{0}: S \rightarrow C^{0}$ defined by

$$
\phi^{0}(p)=\left(\phi_{1}^{0}(p), \cdots, \phi_{g}^{0}(p)\right) \text { where } \phi_{i}^{0}(p)=\int_{p_{0}}^{p} \omega_{i} .
$$

One sees immediately that the map is not well defined for it depends on the path of integration. Since any two images of a point can differ only by an integral linear combination of the columns of the period matrix we identify all such points in $\boldsymbol{C}^{o} . \boldsymbol{C}^{\sigma}$ under this indentification is called the Jacobi Variety of $S$ and is denoted by $J(S)$. The map $\phi^{0}$ is now a well defined map from $S \rightarrow J(S)$. The map $\phi^{0}$ is immediately extendable to divisors on $S$. The definition is the natural one. If $\delta=p_{1}^{\alpha} \cdots p_{k}^{\alpha} k$ is a divisor on $S, \phi^{0}(\delta)=\sum_{i=1}^{k} \alpha_{i} \phi^{0}\left(p_{i}\right)$.

We now consider $\theta\left(\phi^{0}(p), \Pi\right)$ or more generally $\theta\left(\phi^{0}(p)-e, \Pi\right)$ for any $e \in C^{a}$. For a modern presentation of the theory of the Riemann theta function the reader should consult [L]. The properties of the theta function that we shall need are the following: Either $\theta\left(\phi^{0}(p)-e, \Pi\right)$ is identically equal to zero on $S$ or else $\theta\left(\phi^{0}(p)-e, \Pi\right)$ has precisely $g$ zeros on $S, p_{1} \cdots p_{g}$. In the latter case denoting the divisor of zeros by $\delta$ we have $\phi^{0}(\delta)+K^{0} \equiv e$ where $K^{0}$ is a vector of constants in $C^{0}$ depending only on $p_{0}$ and $\Gamma, \Delta$, and $\equiv$ means equality in $J(S)$. Another property that we shall need is that $\theta(e)=0$ if and only if there is an integral divisor $\Delta$ on $S$ of degree $g-1$ and $e \equiv \phi^{0}(\Delta)$ $+K^{0}$ for some point $p_{0} \in S$. Actually, it is shown in [L] that if $e \equiv \phi^{0}(\Delta)+K^{0}$ then $e \equiv \phi^{i}(\Delta)+K^{i}$ where the superscript $i$ means that a point $p_{i}$ has been chosen in the definition of the map $\phi$ from $S \rightarrow J(S)$. Finally we remark that the order of vanishing of $\theta$ at $e$ is given by $i(\Delta)=\operatorname{dim} \Omega(\Delta)$ and $\Omega(\Delta)=\left\{\omega \in A_{1}\right.$ such that the divisor of $\omega$ is a multiple of $\Delta\}$. 
THEOREM 1. Let $\delta=p_{1} \cdots p_{g-1}$ be any integral divisor of degree $g-1$ on $S$ with the property that $i(\delta)=1$. Let $\omega \in \Omega(\delta)$. Then $\omega=\lambda \sum_{j=1}^{g}(\partial \theta / \partial \zeta)(-e) \omega_{g}$ where $e \equiv \phi(\delta)+K$.

Note. In the final statement of the theorem we have $e \equiv \phi(\delta)+K$ without any superscript on $\phi$ or $K$. This is all right since $\delta$ is an integral divisor of degree $g-1$ and hence $e$ is determined independent of the base point in the definition of $\phi$.

ProOF. We shall first prove this theorem under the assumption that $p_{i} \neq p_{j}$ for $i \neq j$ which is precisely Theorem 12 in [L]. Since $i(\delta)=1$, the space $\Omega(\delta)$ is 1 -dimensional and hence there is a unique integral divisor $\delta^{\prime}$ of degree $g-1$ such that $i\left(\delta \delta^{\prime}\right)=1$. Therefore $\delta \delta^{\prime}$ is the divisor of an $\omega \in A_{1}$ and we claim that $\omega=\lambda \sum_{j=1}^{o}\left(\partial \theta / \partial \zeta_{j}\right)(-e) \omega_{j}$ where $e \equiv \phi(\delta)+K$. To show that this is actually the case, consider $\theta\left(\phi^{0}(p)-e, \pi\right)$. By the Riemann vanishing theorem [L, Theorem 8] we have $\theta\left(\phi^{0}(p)-e, \pi\right) \neq 0$ on $S$ and hence $\left(\partial \theta / \partial \zeta_{j}\right)(-e) \neq 0$ for some $j=1, \cdots, g$. Therefore $\omega$ is not the zero differential. We show that the divisor of $\omega \in \Omega(\delta)$ ie: $\omega$ vanishes at $p_{i}, i=1, \cdots, g-1$.

To this end let $p_{i}$ be a point in $\delta$. Then $e \equiv \phi^{i}(\delta)+K^{i}$ and $e \equiv \phi^{i}\left(p_{i}\right)$ $+\phi^{i}(\delta)+K^{i}$ since $\phi^{i}\left(p_{i}\right) \equiv 0$. Consider now $\theta\left(\phi^{i}(p)-e, \pi\right)$. There are two possibilities. Either $\theta\left(\phi^{i}(p)-e, \pi\right) \equiv 0$ on $S$ or else $\theta\left(\phi^{i}(p)-e, \pi\right)$ has precisely $g$ zeros on $S$ with a double zero at $p_{i}$. In the former case we simply expand $\theta\left(\phi^{i}(p)-e\right)$ in a Taylor series in a local parameter $Z$ at $p_{i}$ and obtain

$$
\theta\left(\phi^{i}(p)-e, \pi\right)=\theta(-e)+\left(\sum_{j=1}^{g}\left(\frac{\partial \theta}{\partial \zeta_{j}}(-e) \omega_{j}\left(p_{i}\right)\right) Z+O\left(|z|^{2}\right)\right)
$$

If $\theta\left(\phi^{i}(p)-e, \pi\right) \equiv 0$, all coefficients of the expansion vanish and hence in particular

$$
\sum_{j=1}^{g} \frac{\partial \theta}{\partial \zeta_{j}}(-e) \omega_{j}\left(p_{i}\right)=0
$$

In the latter case since $\theta\left(\phi^{i}(p)-e, \pi\right)$ has a double zero at $p_{i}$ the coefficient of $Z$ surely vanishes giving us the desired result. We remark that what has been shown here is simply that $\omega\left(p_{i}\right)=0$ for $p_{i} \in \delta$. This in no way depended on the fact that $p_{i} \neq p_{j}$ for $i \neq j$. The condition of $p_{i} \neq p_{j}$ for $i \neq j$ however does tell us that the divisor of $\omega$ is actually a multiple of $\delta$. If $p_{i}=p_{j}$ for $i \neq j$ the preceding argument would not guarantee that $\omega$ have a multiple zero at $p_{i}$.

In order to remove the restriction of $p_{i} \neq p_{j}$ for $i \neq j$ we consider an arbitrary integral divisor of degree $g-1, \zeta=p_{i}^{k_{1}} \cdots p_{r}^{k_{r}}, i(\zeta)=1$. If 
$e \equiv \phi(\zeta)+K$ and $\omega=\sum\left(\partial \theta / \partial \zeta_{j}\right)(-e) \omega_{j}$ we would like to show that the divisor of $\omega$ is a multiple of $\zeta$. We may view $\zeta$ as a point in $S_{g-1}$, the $g-1$ fold symmetric product of $S$ with itself. In any neighborhood of $\zeta \in S_{g-1}$ we can find points $p_{1}^{\prime} \cdots p_{g-1}^{\prime}$ with $p_{i} \neq p_{j}$ for $i \neq j$ and $i\left(p_{1}^{\prime} \cdots p_{0=1}^{\prime}\right)=1$. Letting $e^{\prime}=\phi\left(p_{1}^{\prime} \cdots p_{0}^{\prime}\right)+K$ by our previous result we know that the divisor of $\omega^{\prime}=\sum\left(\partial \theta / \partial \zeta_{j}\right)\left(-e^{\prime}\right) \omega_{j}$ is a multiple of $p_{1}^{\prime} \cdots p_{o-1}^{\prime}$. Choosing therefore a sequence of points $\zeta^{\prime} \in S_{o-1}$ converging to $\zeta$ we have $\omega^{\prime}$ converging to $\omega$ and this yields the result.

II. In this section we wish to apply the result of the previous section to establish the existence of a basis for $A_{1}$ each of whose elements is a differential with double zeros. In order to do this we shall define a more general theta function than we have used till this point.

Definition 1. The first order Riemann theta function with characteristic

$$
\left[\begin{array}{c}
\epsilon \\
\epsilon^{\prime}
\end{array}\right]=\left[\begin{array}{c}
\epsilon_{1}, \cdots, \epsilon_{o} \\
\epsilon_{1}^{\prime}, \cdots, \epsilon_{g}^{\prime}
\end{array}\right]
$$

is defined by the following series which converges absolutely and uniformly on compact subsets of $\boldsymbol{C}^{\sigma} \times \widetilde{S}_{\boldsymbol{g}}$.

$$
\begin{aligned}
\theta\left[\begin{array}{c}
\epsilon \\
\epsilon^{\prime}
\end{array}\right](\zeta, \Pi)=\sum_{N \in Z^{0}} \exp 2 \pi i\left[\frac{1}{2}\left(N+\frac{\epsilon}{2}\right) \pi\left(N+\frac{\epsilon}{2}\right)\right. \\
\left.+\left(N+\frac{\epsilon}{2}\right)\left(\zeta+\frac{\epsilon^{\prime}}{2}\right)\right]
\end{aligned}
$$

where $\left[\epsilon^{\prime}\right]$ is a $2 \times g$ matrix of integers.

The function $\theta\left[{ }_{\epsilon^{\prime}}^{\circ}\right](\zeta$, II $)$ is an even or odd function of $\zeta$ according to $\epsilon \cdot \epsilon^{\prime} \equiv 0$ or $1 \bmod 2$. The reader will observe that the Riemann theta function defined in the previous section is $\theta\left[\begin{array}{l}0 \\ 0\end{array}\right](\zeta, \Pi)$. Once again for a survey of the theory of theta functions with characteristics the reader is referred to [F2].

Definition 2. A half period in $J(S)$ is a point $e \in J(S)$ such that $2 e \equiv 0$.

It is therefore clear that each half period in $J(S)$ can be written as $\frac{1}{2}\left[\epsilon_{1}^{\prime} e^{(1)}+\cdots+\epsilon_{0}^{\prime} e^{(\theta)}+\epsilon_{1} \Pi^{(1)}+\cdots+\epsilon_{g} \Pi^{(o)}\right]$ where $e^{(i)}$ is the $i$ th column of the $g \times g$ identity matrix $I$ and $\Pi^{(i)}$ is the $i$ th column of $\Pi$. We shall denote the above half period by

$$
\left(\begin{array}{l}
\epsilon \\
\epsilon^{\prime}
\end{array}\right)=\left(\begin{array}{c}
\epsilon_{1}, \cdots, \epsilon_{g} \\
\epsilon_{1}^{\prime}, \cdots, \epsilon_{0}^{\prime}
\end{array}\right) .
$$


Adopting this notation it is clear that $\left.\theta\left[\epsilon_{\epsilon^{\prime}}^{e}\right](\zeta, \Pi)=E(\zeta, \Pi) \theta\left(\zeta+\left(e_{e^{\prime}}^{e}\right)\right), \Pi\right)$ where $E$ is some exponential factor. Hence when one is only interested in the zeros of the theta functions it suffices to simply study the theta function with characteristic $\left[\begin{array}{l}0 \\ 0\end{array}\right]$ and its translates.

In particular let us consider now an odd theta function $\theta\left[e_{e^{\prime}}\right](\zeta, \Pi)$. Clearly $\theta\left[\begin{array}{c}e^{\prime} \\ \epsilon^{\prime}\end{array}\right](0, \Pi)=0$ since an odd function vanishes at the origin. By our above remarks however, we also have that $\theta\left(\left(e_{e^{\prime}}^{e}\right), \Pi\right)=0$ for $\left(\begin{array}{c}\epsilon^{\prime} \\ \epsilon^{\prime}\end{array}\right)$ on odd half period. Oddness or evenness of a half period $\left(\begin{array}{l}e^{\prime} \\ \epsilon^{\prime}\end{array}\right)$ is determined by $\epsilon \cdot \epsilon^{\prime}$.

It therefore follows that for each odd half period $\left({ }_{\epsilon^{\prime}}^{\circ}\right)$ we have $\theta\left(\left(e_{e^{\prime}}^{e^{\prime}}\right), \Pi\right)=0$ and therefore by the remarks in $\S I$ we have $\left(\epsilon^{\prime}\right) \equiv \phi(\delta)+K, \delta$ an integral divisor of degree $g-1$ on $S$. Furthermore, in [F1] it is shown that except for a set of positive codimension in the Torelli space $i(\delta)=1$. We finally remark that for any odd half period $\left(\epsilon^{\prime}\right)$ and associated divisor $\delta$ we have $\delta^{2}$ is the divisor of an element of $A_{1}$.

Theorem 2. Let $\left(e_{e^{\prime}}^{(}\right)_{1}, \cdots,\left(e^{(}\right)_{0}$ be any $g$ odd half periods with the property that $\left(\begin{array}{l}e^{6} \\ e^{\prime}\end{array}\right)$ has a $\left(\begin{array}{l}1 \\ 1\end{array}\right)$ in the ith column and no other $\left(\begin{array}{l}1 \\ 1\end{array}\right)$ columns. Let

$$
\phi_{i}=\sum_{j=1}^{o} \frac{\partial \theta}{\partial \zeta_{j}}\left(-\left(\begin{array}{l}
\epsilon \\
\epsilon^{\prime}
\end{array}\right)_{i} \Pi\right) \omega_{j} .
$$

Then except for a set with positive codimension in the Torelli space the $\phi_{i}$ are linearly independent, hence a basis for $A_{1}$ and each $\phi_{i}$ is a differential with double zeros.

Proof. Since $\theta\left(\left(_{\epsilon^{\prime}}\right)_{i}, \Pi\right)=0$ for each $i$ we have that $\left(\left(_{\epsilon^{\prime}}\right)_{i} \equiv \phi\left(\delta_{i}\right)+K\right.$ and the results of $[\mathrm{F} 1]$ give that except for a set of positive codimension in Torelli space $i\left(\delta_{i}\right)=1$. Hence we can apply Theorem 1 and obtain

$$
\phi_{i}=\sum_{j=1}^{g} \frac{\partial \theta}{\partial \zeta_{j}}\left(-\left(\begin{array}{c}
\epsilon \\
\epsilon^{\prime}
\end{array}\right)_{i}, \Pi\right) \omega_{j}
$$

By Theorem 1 the divisor of $\phi_{i}$ is a multiple of $\delta_{i}$ and since $i\left(\delta_{i}\right)=1$, $\phi_{i}$ is a basis for $\Omega\left(\delta_{i}\right)$. We know however that $\delta_{i}^{2}$ is the divisor of some element of $A_{1}$ [F2]. Therefore, the divisor of $\phi_{i}$ is $\delta_{i}^{2}$ and hence each $\phi_{i}$ is a differential with double zeros. It remains to show that $\phi_{i}, i=1, \cdots, g$, are linearly independent.

Clearly, the differentials $\phi_{i}, i=1, \cdots, g$, are linearly independent if and only if the matrix 


$$
\left(\frac{\partial \theta\left(-\left(\begin{array}{l}
\epsilon \\
\epsilon^{\prime}
\end{array}\right)_{i}, \Pi\right)}{\partial \zeta_{j}}\right), \quad i, j=1, \cdots, g,
$$

is nonsingular or what is of course the same thing

$$
\operatorname{det}\left(\frac{\partial \theta\left(-\left(\begin{array}{c}
\epsilon \\
\epsilon^{\prime}
\end{array}\right)_{i}, \Pi\right)}{\partial \zeta_{j}}\right) \neq 0 .
$$

Since $\operatorname{det}\left(\partial \theta\left(-\left(\epsilon_{\epsilon^{\prime}}^{\epsilon}\right)_{i}, \Pi\right) / \partial \zeta_{j}\right)$ is a holomorphic function on $\mathfrak{S}_{g}$ and also on the subset of $\widetilde{S}_{g}$ which may be identified with the Torelli space it suffices to prove that the determinant is not identically zero on the Torelli space. For, since the determinant is not identically zero its zero set has positive codimension which is of course the statement of the theorem. It therefore suffices to find a point in Torelli space such that at that point

$$
\operatorname{det}\left(\frac{\partial \theta\left(-\left(\begin{array}{c}
\epsilon \\
\epsilon^{\prime}
\end{array}\right)_{i}, \Pi\right)}{\partial \zeta_{j}}\right) \neq 0 .
$$

In order to find such a point we consider a point $\Pi_{0} \in \mathcal{S}_{\text {g }}$ which is a diagonal matrix with diagonal entries $\Pi_{11}, \cdots, \Pi_{o g}$. From the definition of the theta function one sees immediately that

$$
\theta\left(\zeta_{1}, \cdots, \zeta_{g}, \Pi_{0}\right)=\prod_{j=1}^{o} \theta\left(\zeta_{j}, \Pi_{j j}\right)
$$

where each factor of the product is a theta function with $g=1$, and hence

$$
\frac{\partial \theta\left(\zeta_{1}, \cdots, \zeta_{\theta}, \Pi_{0}\right)}{\partial \zeta_{k}}=\frac{\partial \theta}{\partial \zeta_{k}}\left(\zeta_{k}, \Pi_{k k}\right) \frac{1}{\theta\left(\zeta_{k}, \Pi_{k k}\right)} \prod_{j=1}^{o} \theta\left(\zeta_{j}, \Pi_{j j}\right) .
$$

We shall now show that

$$
\operatorname{det}\left(\frac{\partial \theta\left(-\left(\begin{array}{l}
\epsilon \\
\epsilon^{\prime}
\end{array}\right)_{i}, \Pi_{0}\right)}{\partial \zeta_{j}}\right) \neq 0 .
$$

Using the definition of $\left({ }^{\circ}\right)_{i}$ and the preceding remarks we see immediately that 


$$
\begin{aligned}
& \frac{\partial \theta\left(-\left(\begin{array}{l}
\epsilon \\
\epsilon^{\prime}
\end{array}\right)_{i}, \Pi_{0}\right)}{\partial \zeta_{j}} \\
& =\frac{\partial \theta\left(-\left(\begin{array}{l}
\epsilon_{j} \\
\epsilon_{j^{\prime}}
\end{array}\right)_{i}, \Pi_{j^{\prime}}\right)}{\partial \zeta_{j}} \frac{1}{\theta\left(-\left(\begin{array}{c}
\epsilon_{j} \\
\epsilon_{j^{\prime}}
\end{array}\right)_{i}, \Pi_{j j}\right)} \prod_{l=1}^{0} \theta\left(-\left(\begin{array}{c}
\epsilon_{l} \\
\epsilon_{l^{\prime}}
\end{array}\right)_{i}, \Pi_{l l}\right)
\end{aligned}
$$

and hence unless $i=j$ we have $\theta\left(-\left(\begin{array}{l}\epsilon_{i} \\ \epsilon_{i^{\prime}}\end{array}\right), \Pi_{i i}\right)$ appearing in the product and this term in zero since $\left(\begin{array}{l}\epsilon_{i} \\ \epsilon_{i^{\prime}}\end{array}\right)_{i}=\left(\begin{array}{l}1 \\ 1\end{array}\right)$ which is an odd half period for the theta function $\theta\left(\zeta, \Pi_{i i}\right)$. It is therefore clear that the matrix in question is a diagonal matrix with diagonal entries

$$
\begin{aligned}
\frac{\partial \theta\left(-\left(\begin{array}{l}
\epsilon \\
\epsilon^{\prime}
\end{array}\right)_{k}, \Pi_{0}\right)}{\partial \zeta_{k}} \\
=\frac{\partial \theta\left(-\left(\begin{array}{l}
1 \\
1
\end{array}\right), \Pi_{k k}\right)}{\partial \zeta_{k}} \frac{1}{\theta\left(-\left(\begin{array}{l}
1 \\
1
\end{array}\right), \Pi_{k k}\right)} \prod_{l=1}^{o} \theta\left(-\left(\begin{array}{c}
\epsilon_{l} \\
\epsilon_{l}
\end{array}\right)_{k}, \Pi_{l l}\right)
\end{aligned}
$$

which equals

$$
-\frac{\partial \theta\left(\left(\begin{array}{l}
1 \\
1
\end{array}\right), \Pi_{k k}\right)}{\partial \zeta} \frac{1}{\theta\left(\left(\begin{array}{l}
1 \\
1
\end{array}\right), \Pi_{k k}\right)} \prod_{l=1}^{0} \theta\left(\left(\begin{array}{l}
\epsilon_{l} \\
\epsilon_{l^{\prime}}
\end{array}\right)_{k}, \Pi_{l l}\right)
$$

where the last equality follows from the evenness of the theta function. By construction all the terms in the product which do not get cancelled are theta functions of one variable evaluated at even half periods. It is well known that the only zeros of a theta function of one variable occur at the odd half period hence none of those terms vanish. The only remaining thing to be checked is that $\partial \theta\left(\left(\begin{array}{l}1 \\ 1\end{array}\right), \Pi_{k k}\right) / \partial \zeta_{k} \neq 0$, for then since no term on the diagonal vanishes the determinant does not vanish. We observe however that

$$
\theta\left[\begin{array}{l}
1 \\
1
\end{array}\right]\left(\zeta, \Pi_{k k}\right)=\exp 2 \pi i\left[\frac{\Pi_{k k}}{8}+\frac{\zeta}{2}+\frac{1}{4}\right] \theta\left(\zeta+\left(\begin{array}{l}
1 \\
1
\end{array}\right), \Pi_{k k}\right) .
$$


(See remarks after Definition 2.) Therefore,

$$
\begin{aligned}
\frac{\partial \theta\left[\begin{array}{l}
1 \\
1
\end{array}\right]\left(\zeta, \Pi_{k k}\right)}{\partial \zeta}= & \exp 2 \pi i\left[\frac{\Pi_{k k}}{8}+\frac{\zeta}{2}+\frac{1}{4}\right] \\
& \cdot\left[\frac{\partial \theta\left(\zeta+\left(\begin{array}{l}
1 \\
1
\end{array}\right), \Pi_{k k}\right)}{\partial \zeta}+\pi i \theta\left(\zeta+\left(\begin{array}{l}
1 \\
1
\end{array}\right), \Pi_{k k}\right)\right]
\end{aligned}
$$

and in particular we have

$$
\frac{\partial \theta\left[\begin{array}{l}
1 \\
1
\end{array}\right]\left(0, \Pi_{k k}\right)}{\partial \zeta}=\exp 2 \pi i\left[\frac{\Pi_{k k}}{8}+\frac{1}{4}\right] \frac{\partial \theta\left(\left(\begin{array}{l}
1 \\
1
\end{array}\right), \Pi_{k k}\right)}{\partial \zeta} .
$$

We finally remark that $\partial \theta\left[\begin{array}{l}1 \\ 1\end{array}\right](0, \Pi) / \partial \zeta$ is known to be nonzero for any $\Pi \in \widetilde{\Im}_{1}\left[\right.$ SG, p. 268] and hence we have $\partial \theta\left(\left(\begin{array}{l}1 \\ 1\end{array}\right), \Pi_{k k}\right) / \partial \zeta \neq 0$.

We have therefore shown that the determinant in question is not zero at $\Pi_{0}$ a diagonal matrix. Since every neighborhood of a diagonal matrix contains points of the Torelli space, we have by continuity, that the determinant in question does not vanish for some $\Pi$ in the Torelli space and by our initial remarks the theorem is proven.

Finally we remark that the same methods yield a proof of the fact that the vanishing of an even theta constant is special in the sense of moduli (see [F1]).

The same procedure used here will show that $\theta\left[\begin{array}{l}0 \\ 0\end{array}:_{0}^{0}\right][(0, \Pi)$ is not zero in a neighborhood of a diagonal matrix in $\mathfrak{S}_{g}$. Hence $\theta\left[\begin{array}{lll}0 & \cdots & 0 \\ 0 & \cdots & 0\end{array}\right](0, \Pi)$ is not identically zero on $\widetilde{S}_{\rho}$ and therefore one gets the result that the vanishing is special in the sense of moduli.

Since one can go from any even theta constant to the above theta constant by linear transformation the result is established in general.

\section{REFERENCES}

[F1]. Hershel M. Farkas, Special divisors and analytic subloci of Teichmueller space, Amer. J. Math. 88 (1966), 881-901. MR 35 \#4406.

[F2]. - On the Schottky relation and its generalization to arbitrary genus, Ann. of Math. (to appear).

[L]. Joseph Lewittes, Riemann surfaces and the theta function, Acta Math. 111 (1964), 37-61. MR 28 \#206.

[R]. Harry E. Rauch, Functional independence of theta constants, Bull. Amer. Math. Soc. 74 (1968), 633-638. MR 37 \#1590.

[SG]. G. Sansone and J. Gerretsen, Lectures on the theory of functions of a complex variable, Noordhoff Groningen, 1960. MR 22 \#4819.

State University of New York, Stony Brook, New York 11790 